\title{
PARAMETRIC ARCHITECTURAL DESIGN BASED ON OPTIMIZATION ALGORITHM
}

\author{
Zhao Liang1,2, Wang Wenshun ${ }^{1}$ \\ ${ }^{1}$ China University of Mining and Technology, China \\ 2Jiangsu Vocational Institute of Architectural Technology, China \\ *Corresponding Author Email: caitay@aol.com
}

This is an open access article distributed under the Creative Commons Attribution License, which permits unrestricted use, distribution, and reproduction in any medium, provided the original work is properly cited

\section{ARTICLE DETAILS}

\section{Article History:}

Received 1 February 2019 Accepted 19 March 2018 Available online 21 March 2019

\begin{abstract}
As a widely applied method in many fields, parametric design is now finally exerting influences on architectural industry with the rapid development of Internet technology. For this reason, parametric architectural design is studied in this research based on optimization method. First, parametric design and optimization algorithm are introduced; then, the process of modeling and optimization procedures is discussed; at last, the parametric optimization design is applied to solve the complex architectural design problems. Parametric method will thoroughly affect architectural industry. Therefore, architectural designs should be based on optimization algorithm using parametric design to improve the progress and quality of architectural design.
\end{abstract}

\section{KEYWORDS}

Optimization algorithm, parametric design, architectural design

\section{INTRODUCTION}

Around the 1990s, the development of Internet and computer technology led to subtle changes in architectural designs. Computer mastery of complex three-dimensional figures is becoming stronger and stronger. Architects started to consider the possibility of applying parametric design method to architectural designs [1]. It is well-known that there are many contradictions in architectural designs. Architects need to unite these contradictions to form a state of balance to make the finial designs optimal. From this perspective, the combination of parametric design and architectural design is quite natural and perfect. Since parametric designs based on optimization algorithm can be widely applied to varied industries (e.g. aerospace engineering, shipping manufacturing, and logistics transportation), why can't it be used in architectural designs? Objectively, architectural design is not a kind of first-level subject; it is formed under the mature background of various social conditions as a product of the comprehensive development of multiple subjects [2]. The delay of the development process of architectural designs can be explained. Since we have recognized the delay of parametric design's application in architectural industry, changes should be made to catch up the development process. Parametric architectural design based on optimization algorithm is studied in this research under the consideration of the above reasons, aiming to providing useful reference for the designs and research of related areas.

\section{CONCEPTION OF PARAMETRIC DESIGN AND OPTIMIZATION ALGORITHM}

\subsection{Parametric design}

There are many factors in architectural designs. These factors can be integrated in line with the formation idea of functions and be treated as the combination of various parameters. Architectural designs include many requirements from the external and internal environment. The direction and general shape of architectural designs are controlled by these requirements, which are regarded as control parameters [3]. Certain connection exists between the above parameters and control parameters. Some logic relations or mathematical algorithms can be applied to theoretical analysis; consequently, the above connection can be abstracted to form mathematical relations; this way, commands are created. Describe the above parameter relations using computer language and establish a model with computer software. After all the above steps, the actual data of the parameters can be entered into the computer through software; related commands can be conducted according to the given algorithms. Finally, a prototype based on control parameters can be achieved. This is the conception of parametric design [4]. According to the above definitions, the establishment of control parameter is the most important step in parametric designs. Only in this way can the various contradictory parameters be integrated together during the design process. Consequently, these parameters can be led to an optimal equilibrium point to realize integral optimal design.

However, it is not enough to only define parametric designs. When parametric design first appeared and started to be used in architectural design industry, some inspired architects tried to create new design methods, aiming at meet the more and more complicated requirements for architectural designs in modern society. This trend gradually developed into a new architectural design style, which is now known as parametricism [5].

In fact, architectural design is an important component of an era during the changing times. Architectural designs of previous eras can hardly meet the requirements of a new era as time goes by. The emergence of parametric design is actually a call for new design styles when the architectural design system of previous eras falls into a decline. From the technical methods adopted in parametric architectural designs we can see the reflection of the new era. In-depth utilization of computer science provides parametric architectural designs a reliable foundation in the 
process to solve the complicated contradictions in architectural designs of the new era. In this regard, parametric design method is a product of the era [6]. Therefore, it will certainly change along with different eras. Parametric design methods should continue to self-renew to make the design be suitable for the requirements of the time and be accepted by the public.

\subsection{Optimization algorithm}

Optimization algorithm is a method designed to solve optimization problems. Before the last century, the optimization problems were solved with the differential method and variational method of classicism in western countries. During the Second World War, operational research was generated by war operations in the process of military resource optimal allocation. In operational research, the final target of the entire problem is to reach the optimal result; the solution to the optimal problem is a great change. In the 1960s, computer technology and the latest optimization principle started to be applied to engineering designs; this way, a set of unique theory and specific implementation method was formed, which was named as deterministic method [7]. Actually, random method (e.g. Particle Swarm Optimization and Ant Colony Optimization) is the approach with world-wide significance that can solve most of the complicated practical problems. Genetic Algorithm and Simulated Annealing Algorithm are more suitable for architectural designs. Therefore, these two algorithms are presented in the following sections with specific details.

\subsubsection{Genetic Algorithm}

Genetic algorithm is a kind of optimization algorithm invented by the American Professor John Holland along with his university colleagues and students in the late 1960s. Biological population needs a long period to adapt to the environment during the development process. Optimization algorithm exists in this kind of development process, which is exactly the origin of genetic algorithm. Besides, genetic algorithm also draws lessons from the genetic theory and biological evolution theory of Darwin and Mendel.

Genetic algorithm is formed simulating the biological evolution mechanism, which can be used to establish an artificial model to solve some problems. In genetic algorithm, the optimal solution of each problem will be presented with population. The component of this kind of population is individuals. Each individual is unique, presenting specific external features different from those of other individuals. Meanwhile, the gene of each individual is distinctively unique. The differences in external features are the results of varied genes. In other words, the external features are determined by genes. Therefore, the first step of genetic algorithm is to perceive the essence by examining the appearance. In this step, the external features presented by the individual can be internalized to the perspective of genes. Consequently, the cause of its external features can be found. In computer language, this step is named as coding.

The law of natural evolution suggests that only the fittest can survive from natural selection. Based on this law, genetic algorithm is designed to find out the approximate solution of the problem through step-by-step evolvement. During this process, new individuals are selected in line with the degree of fitness among all individuals. Then, the selected new individuals will be mixed with the individuals produced by random method; this way, a population representing new solution set is created. Continue the above process until an optimal individual is created in the last generation of population. This optimal individual can be used as the optimal solution of the problem.

\subsubsection{Simulated Annealing Algorithm}

Simulated annealing algorithm was first proposed in the 1950s, but it was formally recognized by the public in the 1980 s when Kirkpatrick applied it to solve large-scale optimization problems. In essence, simulated annealing algorithm is different from the general optimization algorithm because it is equipped with random properties. Annealing refers to the process of the internal particles of a heated solid matter gradually accelerate their movements in high-temperature burning and then slow down along with the gradual falling of temperature and eventually reach a state of equilibrium, in which the entire internal energy of the solid matter falls to its minimum. Simulated annealing algorithm is a simulation of the above annealing process, and this is the origin of its name. The realization of this algorithm can be classified into six steps.

Step 1: initialize the conditions; set an adequate initial temperature $\mathrm{T}_{0}$; let $\mathrm{T}=\mathrm{T}_{0}$; define an initial solution $\mathrm{S}_{1}$ and the chain length $\mathrm{L}$, namely, the iteration number of each temperature.

Step 2: repeat the following steps under the condition of current temperature and $\mathrm{k}=1,2, \ldots, \mathrm{L}$,

Step 3: produce a new solution $S_{2}$ in the current random disturbance.

Step 4: calculate the increment of the above new solutions; $\mathrm{df}=\mathrm{f} \quad\left(\mathrm{S}_{2}\right)$-f ( $\left.S_{1}\right)$, in which $\mathrm{f}\left(\mathrm{S}_{1}\right)$ is the evaluation function of $\mathrm{S}_{1}$.

Step 5: if $\mathrm{df}<0$, then, $\mathrm{S}_{2}$ can be accepted as the new current solution, namely, $S_{2}=S_{1}$. On the contrary, the acceptance probability of $S_{2}(\exp (-$ $\mathrm{df} / \mathrm{T}$ )) should be calculated; this way, the random number (rand) evenly distributed in interval $(0,1)$ can be produced. In this case, if exp($\mathrm{d} / \mathrm{T})>$ rand, then, $\mathrm{S}_{2}$ can also be accepted as the new current solution and $\mathrm{S}_{1}=\mathrm{S}_{2}$; if not, retain the current solution $\mathrm{S}_{1}$.

Step 6: when the stop condition is satisfied, output the current solution $S_{1}$ as the optimal solution and stop the program. Normally, the stop condition can be met if the new solutions $\mathrm{S}_{2}$ of several continuous Metropolis chains are not accepted. In this case, the algorithm can be stopped. Another method is to set an end temperature. If the stop condition is not satisfied, return to Step 2 in accordance with attenuation function.

\section{MODELING AND OPTIMIZATION PROGRAMMING PROCESS}

In the resolution of optimization problems, if the parametric method is applied to complicated problems from the start, the user will inevitably fall into a passive position and can hardly escape from it. Hence, users of parametric methods should first try to solve simple optimization problem. In this process, users should pay attention to the rules and then apply these rules to solve complicated practical problems. This way, users can obtain a good capacity for easily applying parametric methods.

The parameterization of simple optimization problem includes the definition of design issues, the parameterized generation of the project, and the evaluation and optimization of the design [8]. To clearly illustrate the modeling and optimization programming steps, the general frameworks are listed as below.

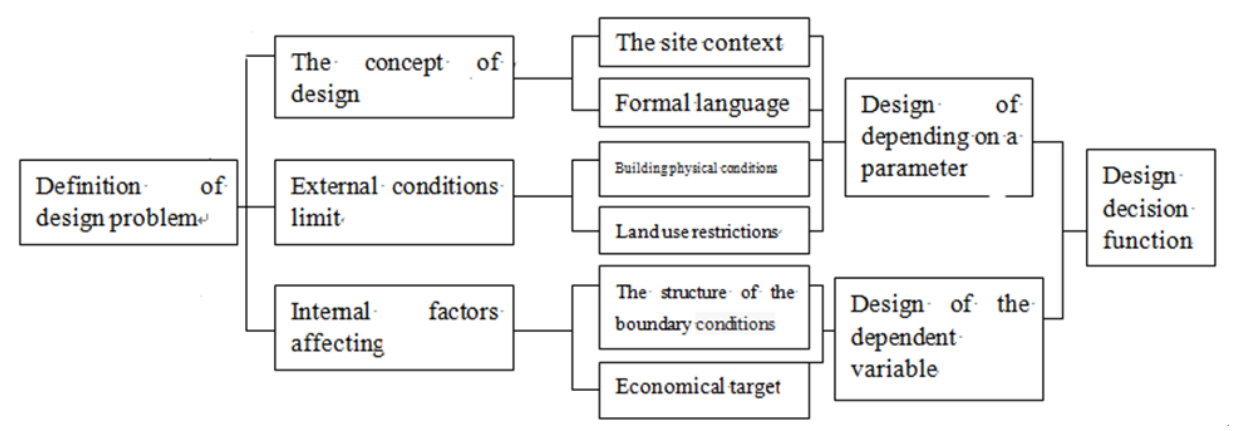

Figure 1: Definition of design problems 


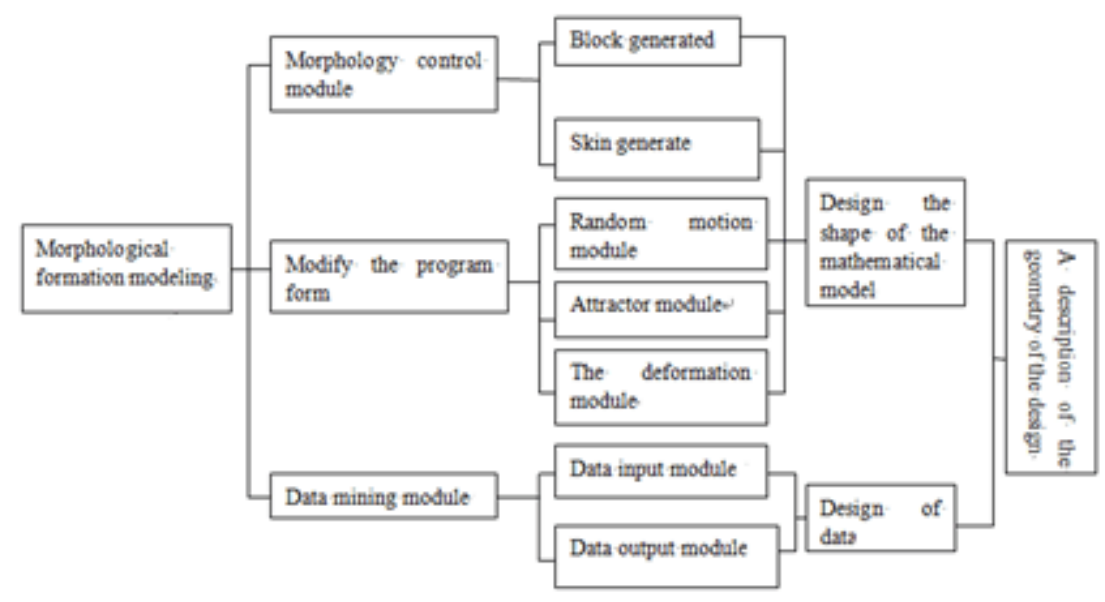

Figure 2: The parameters of the scheme

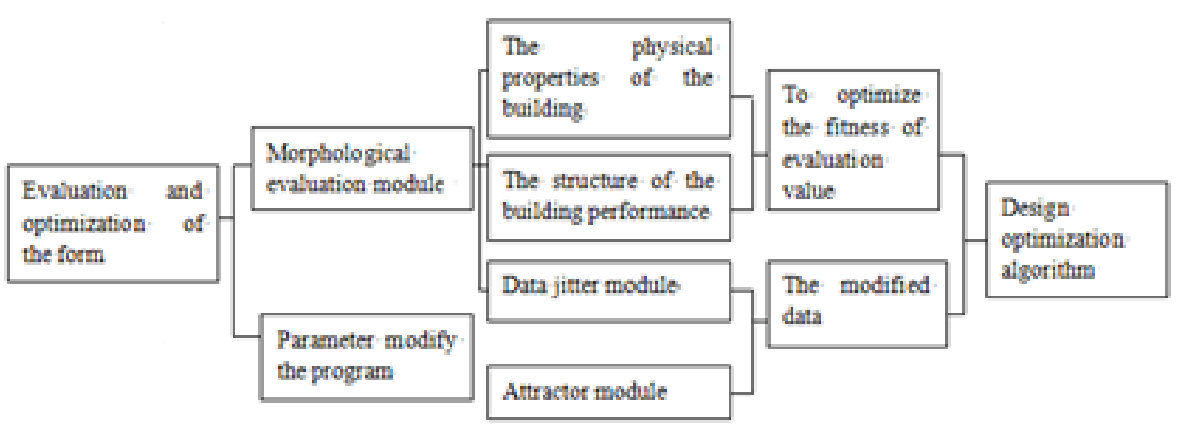

Figure 3: Design evaluation and optimization

\section{PARAMETRIC DESIGN OF COMPLICATED PRACTICAL PROBLEMS}

Practical problems are always complicated. Parametric design is accepted by the public for the reason that the model established with parametric design can approach the practical problem as much as possible. In this research, the architectural design of the National Library of the Republic of Kazakhstan is taken as the example to analyze the application of parametric designs in complicated architectural designs.

\subsection{Definition of design issues}

The design of the National Library of the Republic of Kazakhstan was provided by BIG Architects in 2009; this design won the first-place prize in the competitive bid on the program of this library. The National Library of the Republic of Kazakhstan is located in the capital of Kazakhstan, covering an area of 33,000 square meters. In this design, the infinite changes of space combinations are integrated into a continuous surface. This way, various national symbols are combined together to form a whole. Mobius strip is applied in this design, which has two perfect structures. One is a circle and the other is a spiral public space, forming an interlocking structure. The ring shape makes the library more concise and fresher in appearance. Meanwhile, this shape gives people a never-ending feeling in infinite circulation, indicating that there is no shore in the sea of knowledge.

\subsection{Establishment of the model}

In the design of the National Library of the Republic of Kazakhstan, the key to modeling is to have a profound research and acknowledgement of the Mobius strip-shaped architecture. Analysis reveals that this kind of structure can be created by giving a strip a $180^{\circ}$-twist, and then connecting the ends of the strip to form a loop. In this research, a strip with rectangular cross-section is used to form a Mobius strip model through the above method for further analysis. In this process, Circle arithmetic unit is applied to draw a circular trace formed by multiple splashes on a plane. Besides, Perp Frames arithmetic unit is also utilized to create the reference plane for the twist of the rectangular strip. Detailed procedures are shown in Figure 4.
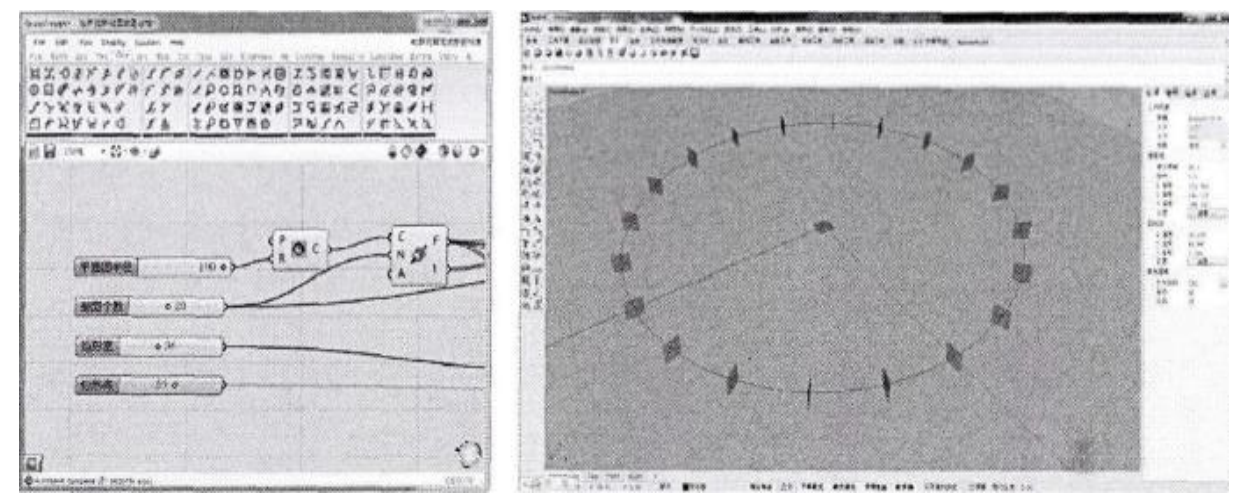

Figure 4: The first stage of modeling 
Next, Number Slide arithmetic unit can be used to control the size of the rectangular cross-section. Build a rectangle; rotate these rectangles in different angles and keep a record of each angle. A data column can be established with Series arithmetic unit to store the values of the above angles. In the end, the data of rectangles, reference plane, and the recorded twisting angles should all be input to the rotate arithmetic unit for computations to obtain the rectangular cross-section.
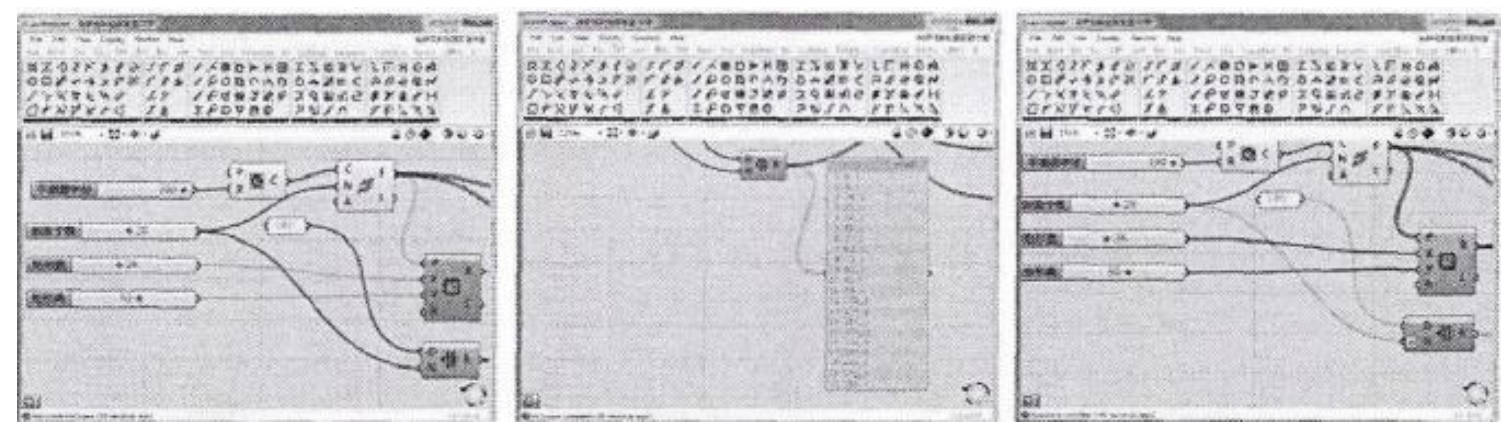

Figure 5: Modeling in the second stage

However, things are not that simple. In practical operations of modeling, the rotating center of the rectangle is one of the endpoints of the rectangle. In this case, the cross-sections of its two ends will be separated. More specifically, these two cross-sections will be in different vertical coordinates. This problem can be solved by finding the midpoint of the rectangle and transferring it to the strip.
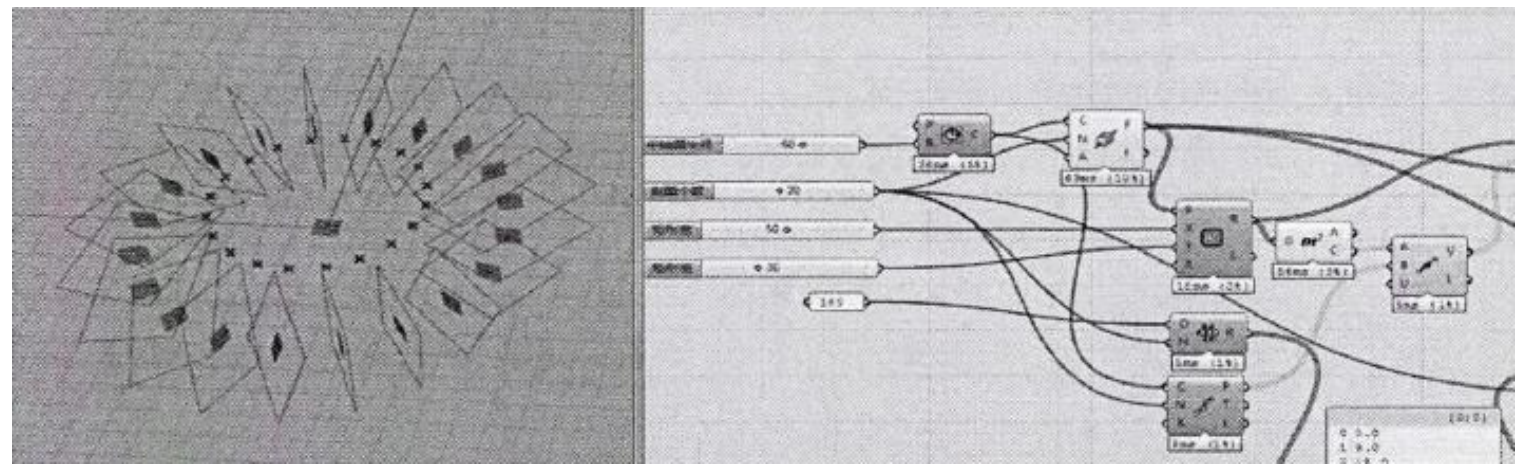

Figure 6: Modeling the third stage

Then, Loft arithmetic unit can be used to transfer the twisted rectangle to form a Mobius strip. But the end-to-end connecting section of the first Mobius strip created in this research was not very smooth and eventually parted, which was totally unexpected. In this research, there are totally 18 rectangles for twisting; all the planes should be twisted except the start plane. For this reason, the above problem can be solved by removing the output data of the last Range arithmetic unit, and then connecting with the rotating angle input end. Furthermore, the rectangle with the largest serial number should be extracted separately with List item arithmetic unit; rotate this rectangle for $180^{\circ}$. Combine the rectangles twisted in previous steps with this twisted rectangle to form a data sheet using Merge arithmetic unit. Connect with Loft arithmetic unit and don't use close lofting; this way, a normal Mobius strip is finally created.

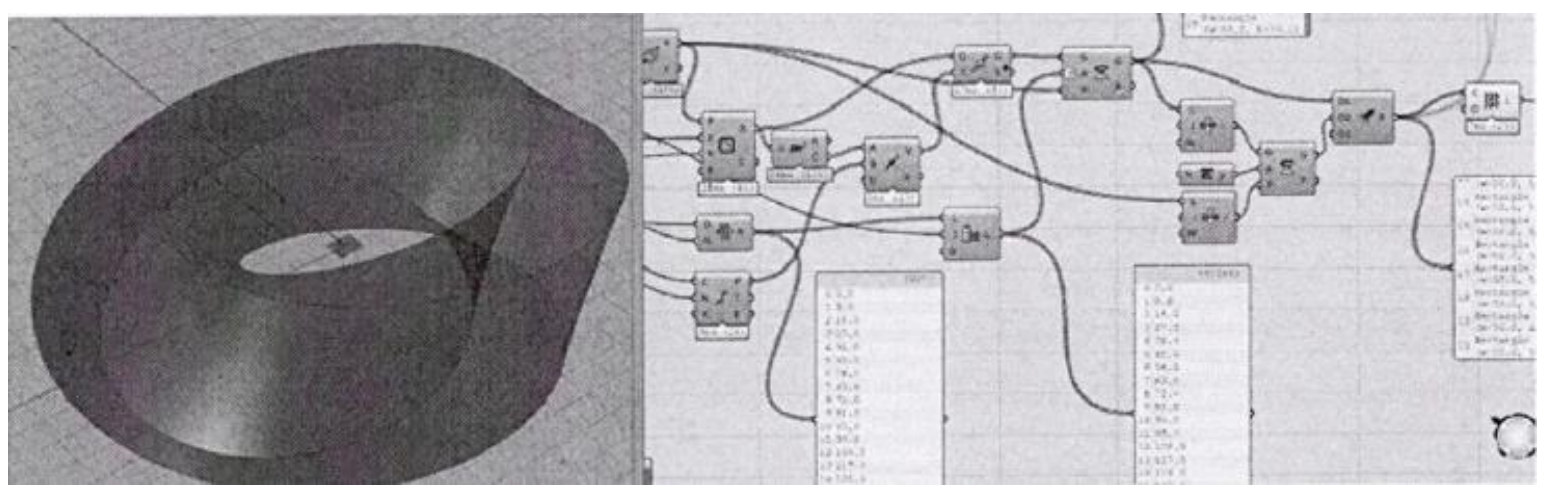

Figure 7: Modeling stage 4

\subsection{Evaluation of the optimization program}

It can be concluded from the above modeling process that there are multiple optimization problems of parametric variables. The optimal solution should be described from two aspects: the value of each parametric variable and the combinations of each parametric variable's parameters. Contradictions between local parts and the whole are likely to emerge during the process of solving the optimal solution. Improper combinations of parameters may lead to local optimization but without global optimization. To solve this problem, some parameters of Galapagos can be changed, and multiple values can be adopted to conduct multiple computations. In this way, the occurrence probability of global optimal solution can be significant increased, which can effectively avoid the confusion caused by local optimal solutions.

Additionally, if the received optimization problem is not very definite, or the optimization process is limited, the optimal solution can be obtained by setting some ideal conditions. This process can help to define the value domain and target value of the optimization search, which can greatly reduce time consumption and be regarded as an optimization of the solution process. 


\section{CONCLUSION}

The parametric method based on optimization algorithm has proved to be concise and effective after years of practical applications. However, it still lacks large-scale applications in architectural design fields. In this research, the parametric architectural design based on optimization algorithm is analyzed with profound theories in simple languages. The findings indicate that parametric model should first be applied to solve simple optimization problems to find the common rules. Then, it can be used to solve the complicated practical problems. The combination of parametric method with optimization algorithm can well solve many and various complicated architectural designs problems, through which the contradictions between the factors of final influencing the final results can be reasonably resolved. In this respect, the parametric method based on optimization algorithm can be popularized in architectural design fields and even other industries.

\section{REFERENCES}

[1] Nowotniak, R., Kucharski, J. 2012. GPU-based tuning of quantuminspired genetic algorithm for a combinatorial optimization problem. Bulletin of the Polish Academy of Sciences Technical Sciences, 60(2), 323330.

[2] Yu, Y.Y., Lin, Y., Chen, M. 2015. A new method for ship inner shell optimization based on parametric technique. International Journal of
Naval Architecture \& Ocean Engineering, 7(1), 142-156.

[3] Geyer, P. 2008. Multidisciplinary grammars supporting design optimization of buildings. Research in Engineering Design, 18(4), 197-216.

[4] Shimoda, M., Liu, Y., Morimoto, T. 2014. Non-parametric free-form optimization method for frame structures. Structural \& Multidisciplinary Optimization, 50(1), 129-146.

[5] Wang, Q., Frank, A.A 2014. Plug-in HEV with CVT: configuration, control, and its concurrent multi-objective optimization by evolutionary algorithm. International Journal of Automotive Technology, 15(1), 103115 .

[6] Rao, R.V., Savsani, V.J. 2012. Design Optimization of Selected Thermal Equipment Using Advanced Optimization Techniques[M]// Mechanical Design Optimization Using Advanced Optimization Techniques. Springer London, 195-229.

[7] Ling, T., Taniar, D., Smith, K. 2002. Parametric Optimization in Data Mining Incorporated with GA-Based Search[M]// Computational Science — ICCS 2002. Springer Berlin Heidelberg, 582-591.

[8] Dai, X., Yuan, X., Wu, L. 2015. A novel harmony search algorithm with gaussian mutation for multi-objective optimization. Soft Computing, 1-19.

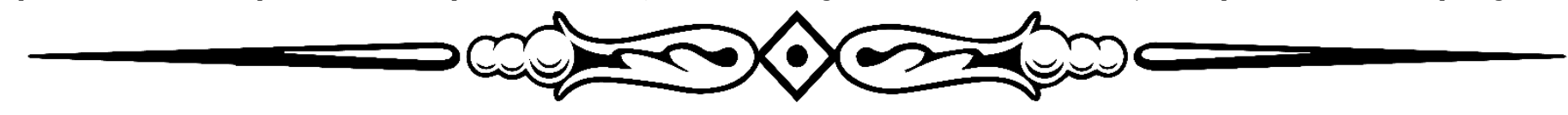

